\title{
THE LYMAN- $\alpha$ IMAGING SOLAR TELESCOPE (LIST) ON THE KOREA SCIENCE AND TECHNOLOGY SATELLITE-2 (STSAT-2)
}

\author{
M. JANG \\ Dept. of Astronomy and Space Science, Kyung Hee University, Yongin, Kyunggi-Do, 449-701, Korea \\ Space Payload Research Center, Kyung Hee University, Yongin, Kyunggi-Do, 449-701, Korea, \\ E-mail: mjang@khu.ac.kr
}

\begin{abstract}
LIST is the Lyman- $\alpha$ Imaging Solar Telescope, a project funded by the Korean government to fly on the second Korean Science and Technology research Satellite (STSat-2) due to launch in December 2005. The Principal Investigator is Dr. Minhwan Jang of Kyung-Hee University and of the Space Payload Research Center (SPARC), a consortium of Korean universities and institutions formed to develop scientific research projects in space. The purpose of the LIST project is to design, build, and operate an instrument on STSat-2 which will make images of the Sun from Earth orbit at the wavelength of the Hydrogen Lyman- $\alpha$ emission line at $121.6 \mathrm{~nm}$. LIST has a simple design concept comprised of a small telescope to image the full disk of the Sun onto a CCD detector and a set of filters to isolate the $121.6 \mathrm{~nm}$ wavelength.
\end{abstract}

Key words : Sun: instrumentation

\section{INTRODUCTION}

The composition, structure, and dynamics of the earth's upper atmosphere is primarily driven by the full-disk solar flux incident on the top of the atmosphere in the vacuum ultraviolet(VUV: $<200 \mathrm{~nm}$ ) wavelength range. The spectral irradiance of of the sun in the VUV varies by a few percents at $200 \mathrm{~nm}$, by over a factor of two at hydrogen Lyman- $\alpha(121.6 \mathrm{~nm})$, and by orders of magnitude at X-ray wavelengths, on time scales of $\sim 11$ years, $\sim 27$ days, and minutes to hours. The $\sim 11$ year solar cycle variability in VUV irradiance is caused by the increased number of active regions on the face of the sun during solar maximum and the virtual absence of such active regions during solar minimum. The $\sim 27$-day variability is produced by the rotation rate of active region across the sun as seen from earth. Also shorter time scale variability due to flares also occurs. Solar photons interact with the atmosphere as a function of their wavelength, with the shorter wavelengths depositing their energy higher in the atmosphere and the longer wavelengths penetrating to lower altitudes. Particularly, Lyman- $\alpha$ at $121.6 \mathrm{~nm}$, the most intense emission line in the solar VUV, can penetrate down to $70 \mathrm{~km}$ in altitude in the Earth's atmosphere where the energy is deposited mainly via dissociation of $\mathrm{O}_{2}$. Lyman- $\alpha$ photons in the mesosphere also ionize nitric oxide and dissociate water, both of which are important in the chemistry of ozone in this region. Understanding the variability of the solar VUV is a critical part of understanding the variability of the terrestrial upper atmosphere. Solar hydrogen Lyman- $\alpha$ emissions originate from a range of altitudes in the solar chromosphere and the transition region between the chromosphere and corona. Because of this, Tobiska (1988) explored the use of Lyman- $\alpha$ as a proxy for modelling the

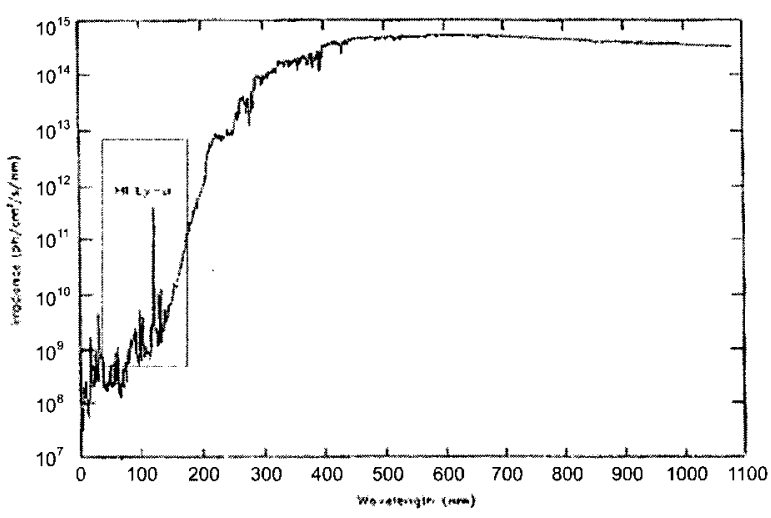

Fig. 1.- The solar spectral irradiance for solar minimum conditions. Data longward of $2000 \mathrm{~nm}$ are from Arvesen et al. (1969), and data shortward of $200 \mathrm{~nm}$ are from Woods \& Rottman (1997).

solar EUV correlations (Tobiska 1990) and subsequent revisions of that model (Tobiska 1991, 1994, 1995; Tobiska et al. 1993; Tobiska \& Eparvier 1997). Partly because solar Lyman- $\alpha$ emissions are optically thick, the Lyman- $\alpha$ irradiance variability behaves somewhat differently than other VUV emissions. Lyman- $\alpha$ is one of the more difficult solar VUV emissions to model. Consequently, the LIST mission will concentrate on the Lyman- $\alpha$ emission. Long term observations of full-disk Lyman- $\alpha$ irradiance have been made by instruments on the Atmospheric Explorer-E(AE-E) satellite, the Solar Mesospheric Explorer (SME) satellite, and the Upper Atmosphere Research Satellite (UARS) (Woods \& Rottman 1997). In addition, several sounding rockets dating back to the 1950s (White 1977) and up through 


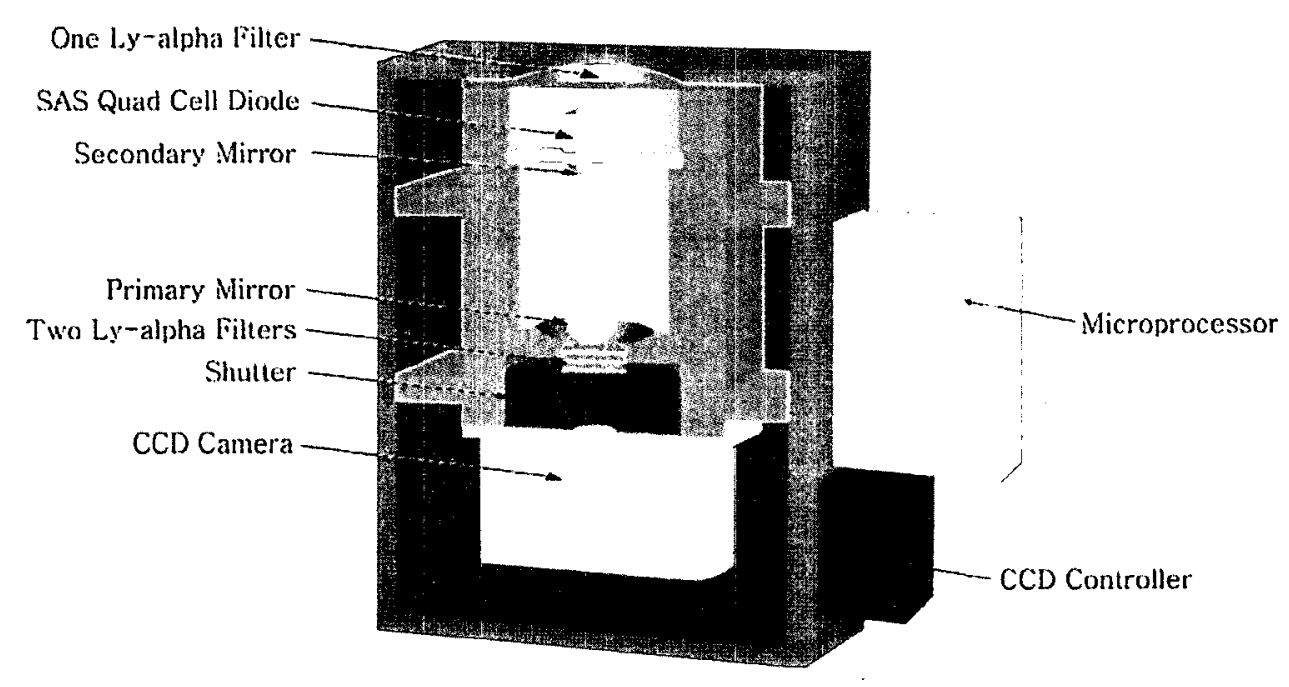

Fig. 2.- A mechanical cross section of the SLIM instrument. The CCD, CCD47-10 s eries, manufactured by E2V, is back-illuminated and two-dimensional with $512 \times 512$ pixels. Each pixel is $24 \mu \mathrm{m}$.

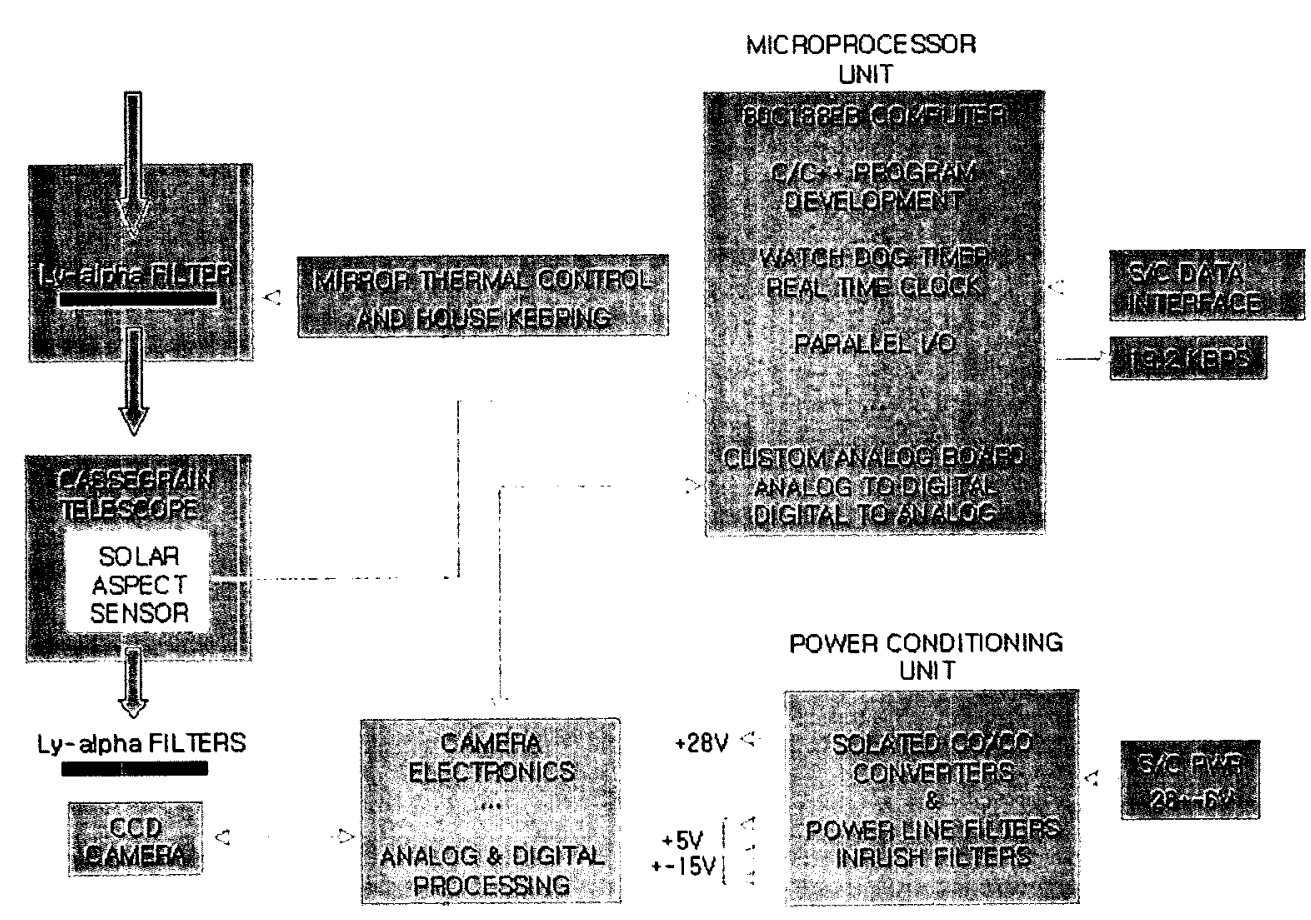

Fig. 3. - Electrical and optical block diagram for LIST 
the present (Eparvier et al. 1997) have measured the Lyman $-\alpha$ irradiance.

Previous full disk Lyman- $\alpha$ images of the Sun have been very interesting and useful scientifically, but have been only five-minute "snapshots" obtained on sounding rocket flights (eg. Bonnet et al. 1982; Foing and Bonnet 1984; Allen et al. 1997). All of these observations to date have been snapshots, with no time resolution to observe changes in the chromospheric structure as a result of the evolving magnetic field, and its effect on the Lyman- $\alpha$ intensity. Therefore, the LIST full disk imager will provide a unique opportunity for the study of the sun in the Lyman- $\alpha$-region with the high time and spatial resolution for the first time.

\section{MISSION OBJECTIVES}

The LIST instrument is a Solar Lyman- $\alpha$ Imager which will answer key questions about the sources of variability in the chromosphere and transition region of the solar atmosphere. Understanding the reasons for solar irradiance variability and the affects of such variability on the Earth's atmosphere are also major themes in NASA's Sun-Earth Connection Program. Hydrogen Lyman- $\alpha$ at $121.6 \mathrm{~nm}$ is one of the brightest emissions from the chromosphere and the transitions region, so it is a natural diagnostic of solar variability in those regions. LIST will be used to study the morphology and evolution of active regions on the Sun which are believed to be the primary cause of solar VUV irradiance variability. The main scientific objectives of the LIST mission are: (1) to understand how the evolution of structures in the chromosphere and transition region of the sun's atmosphere contribute to the variability of solar irradiance at Lyman- $\alpha$ and other VUV wavelengths, (2) to improve models of solar VUV irradiance variability by incorporating plage, enhanced network, active network, and quiet sun indices derived from images of the sun, and (3) to study the affects of solar VUV variability on the terrestrial upper atmosphere. Also there are following 4 sub objectives. (1) Studying the physical properties and processes in the solar active region with Lyman- $\alpha$ images and spectroscopic data. (2) Studying on the variation of the properties of the ULF waves and radio propagation in the earth' magnetosphere by Lyman- $\alpha$. (3) Understanding of the property of radio propagation in the terrestrial upper atmosphere depending on solar activity. (4) Studying of ULF waves in the earth magnetosphere. The LIST mission complements, and also benefits from, solar VUV irradiance measurements from the NASA UARS, TIMED, and EOS missions and solar imaging data at other wavelengths from SOHO, Yohkoh, TRACE, magnetosphere satellites such as POLAR, Geotail and ground-based observatories.

To achieve the scientific goals listed above is to build and fly an earth-orbiting instrument that will make images of the Sun at the hydrogen Lyman- $\alpha$ wavelength on a daily (or more frequent) basis at a resolution of
8 arc-sec or better for a minimum of one year during active solar conditions. These images will be analyzed to retrieve information about the evolution of the solar atmospheric structures that cause VUV irradiance variability. They will be compared with images taken at other wavelengths such as the ground-based CaII K, SOHO EIT images at EUV wavelengths, TRACE FUV images, and Yohkoh and NOAA-GOES soft X-ray images. The images will also be used in conjunction with simultaneous absolute full disk irradiance measurement s made by TIMED-SEE (EUV and FUV), SNOE(soft $\mathrm{x}$-rays), and possibly the SOLSTICE instrument on UARS or EOS (Lyman- $\alpha$, FUV) if the relative timing permits, to calibrate and correlate full-disk irradiance with the variability in the solar atmospheric structure as seen by the images. Current models of solar irradiance variability will be expended and improved upon using the Lyman- $\alpha$ images and the other data sources. In addition, the affects of solar variability on the terrestrial upper atmosphere will be studied by comparing the data from LIST and other sources with the data of thermospheric and mesospheric structure, chemistry, and dynamics through models and measurements

\section{INSTRUMENTATION}

The LIST instrument design is that of a simple CCD camera. It has following major components: (1) interference filters to isolate Lyman- $\alpha$ and sufficiently suppress visible light contamination; (2) a telescope to focus the solar image; (3) a back-illuminated, twodimensional CCD detector; (4) a microprocessor to control the instrument; and (5) a power conditioning unit to filter and distribute power from the spacecraft to the LIST components. A mechanical cross section diagram of LIST showing these components is presented in Figure 2. A block diagram showing the functional interconnections between the LIST components is shown in Figure 3. The pointing and telescope mirrors all will have a $\mathrm{MgF} F_{2}$ coating to enhance reflectivity in the VUV (approximately $70 \%$ at Lyman- $\alpha$ ). The telescope has been designed to put a 45 arc-minute field-of-view on the $\mathrm{CCD}$, giving a minimum resolution of 8 arc-sec/pixel. A Cassegrain design with a concave parabolic primary mirror and a convex hyperbolic secondary mirror was chosen for its compact size.

The narrowband interference filters (Acton Research, Inc.) have a bandpass of approximately several $\mathrm{nm}$ FWHM, centered on $122 \mathrm{~nm}$. The peak Lyman$\alpha$ transmittance is about 0.08 whereas the visible light transmission is on the order of $10^{-5}-10^{-4}$. Because the solar spectral irradiance is approximately three orders of magnitude brighter in the visible than at Lyman$\alpha$, and because the visible irradiance is a continuum whereas solar $\operatorname{Lyman}-\alpha$ is an isolated line emission, a single interference filter will not suffice and it is required to use multiple filters in series to achieve enough visible-to-Lyman- $\alpha$ suppression. LIST will be designed to use a minimum of three interference filters to ensure 


\begin{tabular}{|c|c|c|c|c|c|c|c|c|}
\hline \multirow[b]{2}{*}{ ID } & \multirow{2}{*}{ TITLE } & \multirow{2}{*}{ Start Date } & \multirow{2}{*}{ Final Date } & 2 year & 2003 year & 2004 year & \multirow{2}{*}{\multicolumn{2}{|c|}{2006 year }} \\
\hline & & & & & $01 / 02 / 03 / 04$ & $01102[03104$ & & \\
\hline 1 & LIST Project & $02-10-01$ & $05-12-31$ & & & & & \\
\hline 2 & Stan & $02-10-01$ & $02-10-01$ & & & & & \\
\hline 3 & kick-off & $02-11-14$ & $02-11-14$ & & & & & \\
\hline 4 & System Requinement Review (SRR) & $03-01-14$ & $03-01-14$ & & System Requirem & ents Review (SRR) & & \\
\hline 5 & System Detintion Review (SDR) & 03-03-11 & $03-03-11$ & & Asystem Delinitic & ion Review (SDR) & & \\
\hline 6 & Preliminary Design Review (PDR) & 03-08-01 & 03-08-01 & & APrelimir & inary Design Review & (PDR) & \\
\hline 7 & Critical Design Review (CDR) & 04-05-01 & 04-05-01 & & & ACritical Desi & on Review (CDR) & \\
\hline 8 & PFM Delivery & $01-11-01$ & $01-11-01$ & & . & & M Delivery & \\
\hline 9 & FM Delivery & $05-05-02$ & 05-05-02 & & & & AFM Delivery. & \\
\hline 10 & FTR, LRR & 05-06-01 & 05-06-01 & & & & AFTR, LRR & \\
\hline 11 & Finish & $05-12-31$ & $05-12-31$ & & & & & \\
\hline 12 & Manufacture & 02-10-01 & $05-17-31$ & & & & & \\
\hline 13 & Concept Study & 02-10-01 & $02-10-31$ & & ncept Study & & & \\
\hline 14 & Mission Analysis \& Design & $02-10-01$ & $02-12-31$ & & Mission Analysis \& & Design & & \\
\hline 15 & Requirements Review & $03-01-02$ & 03-03-05 & & 8. Requirements $\mathrm{F}$ & Review & & \\
\hline 16. & Specitication Review & 03-02-04 & $03-06-20$ & & Specificati & ion Review & & \\
\hline 17 & Parts Purchase & 03-05-05 & $05-02-15$ & & 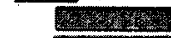 & 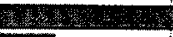 & Parts Purchase & \\
\hline 18 & SMIEM Development & 03-04-30 & $04-04-29$ & & 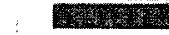 & SM/EM DeV & Fopment & \\
\hline 19 & PFM Development & 04-05-05 & $05-02-20$ & & & का & PFM Developmer & \\
\hline 20 & OTR (PFM) & 05-01-01 & $05-02-20$ & & $\vdots$ & & OTR (PFM) & \\
\hline 211 & FM Development & 05-01-01 & $05-07-31$ & & & & - & elopment \\
\hline 22 & Environmental Test & 04-05-03 & $05-07-31$ & & & & Environme & ental Test \\
\hline
\end{tabular}

Fig. 4.- LIST program schedule

that the images taken by the instrument are Lyman$\alpha$ images, and not contaminated by sunlight at other wavelengths.

A short integration time is beneficial for reducing the solar tracking requirements and reducing the amount of sunlight-induced heating of the optics and detector. Thus we plan to utilize 0.1- 1 second exposure time for the observations. The microprocessor for the LIST, we will use 80188 or 80196 CPU. The duties of the microprocessor will be to: (1) interpret and execute LIST commands, (2) monitor LIST health and safety during observations, (3) package SLIM science and housekeeping data for transfer to the spacecraft telemetry system, and (4) communicate with the spacecraft. To minimize the risk of single event upsets corrupting data, the microprocessor will only be activated for the minimum time before and after a solar observation to allow for solar acquisition, image integration, and transfer of data to the spacecraft. At all other times the microprocessor will be turned off. A signal from the spacecraft will be used to activate the microprocessor.

\section{MISSION DESIGN AND DATA ANAL- YSIS}

The mission duration for LIST will be two years. During two years the sun will undergo approximately 26 revolutions as seen from the earth giving enough time to observe the individual active regions which typically last about 6 months, and variability due to the changing view of active regions on the face of the sun from rotation to rotation. Although the exact inclination of STSAT-2 is not decided yet, its $300 \mathrm{~km}$ to $1500 \mathrm{~km}$ sun-synchronous orbit allow for approximately 1 hour window per orbit for LIST to observe the sun.
We plan to take 18 images per orbit, around $200 \mathrm{im}$ ages per day. There will be 2 observation modes. One is 'quiet sun observing mode' for 8 months per year, another is 'explosive event observing mode' for 4 months period per year.

The images read out from CCD will be transferred digitally to the microprocessor. Then, with housekeeping data, these images will be transferred to the spacecraft for storage until ground contact is made. The spacecraft will store the LIST data and will make twicea-day dumps to the ground station. Commands for LIST will be uploaded to the spacecraft twice-a-day basis except during the instrument checkout phase at the beginning of the mission and at occasional times when adjustments to LIST programming need to be made. LIST raw telemetry will be obtained from Satellite Research Center(KAIST) on a daily basis. The LIST data will be processed at the Space Payload Research Center(Kyung Hee Univ.). All LIST data products, including the raw TM data, will also be archived at the Space Payload Research Center(Kyung Hee Univ.) and accessible to the general public via web pages. Prior to the actual analysis on the same intensity scale by flat-fielding each image, removing the dark count, and then normalizing the intensities of each image to its average background quiet-sun intensity. After this preprocessing, masks of plage, enhanced network, active network, and quiet-sun are computed with an algorithm which uses criteria of size, intensity, filling factor, and contiguity to identify and separate the different solar structures. 


\section{DISCUSSION}

The second Korea Science and Technology Satellite (STSat-2) will be the first Korean satellite which is launched by Korean made launch vehicle(KSLV1). Also STSat-2 will be the first full-scale satellite launched at the Korea Space Center which is now under construction. With the acknowledgement of this historic mission, SPARC team approached the project management very carefully. We focused our effort to define science goals and translate those into clear mission requirements. And we have taken these requirements and evolved the design of the instrumentation to result in a minimum but optimized system. Figure 4 specifies the program schedule. The LIST project is led by a single PI supported by SPARC team which consists of the professors and the graduate students in the related fields from the different Korean universities.

\section{REFERENCES}

Allen, M. J., Oluseyi, H. M., Walker, A. B. C., Hoover, R. B., \& Barbee, T. W. 1997, Chromospheric and coronal structure of polar plumes, I. Magnetic structure and radiative energy balance, Sol. Phys., 174, 367

Arvesen, J. C., Griffin, Jr., and Pearson, B. D. 1969, Determination of extraterr estrial solar spectral irradiance from a resear aircraft, Appl. Optics, 8, 2215

Bonnet, R. M. et al. 1982, Rocket photographs of fine structure and wave patterns in the solar temperature minimum, A\&A, 111, 125

Foing, B., \& Bonnet, R.M. 1984, Results from the Transition Region Camera, Adv. Space Res., 443

Eparvier, F. G. et al. 1997, Solar minimum spectral irradiance from the soft $\mathrm{x}$-rays to the FUV, Eos, Trans., AGU, 78, F560

Tobiska, W.K. 1988, A solar extreme ultraviolet flux model, Ph.D. dissertation, Univ. Colorado, Boulder

Tobiska, W.K. 1990, A solar extreme ultraviolet flux model, Contribution 9, Ear th and Planets. Atmos. Group, Space Sci. Lab., Univ. Calif., Berkely

Tobiska, W.K. 1991, Revised solar extreme ultraviolet flux model, J. Atmos. Ter r. Phys., 53, 1005

Tobiska, W. K. 1994, Modeled soft x-ray solar irradiances, Sol. Phys., 152, 207

Tobiska, W.K. 1995, Current status of solar EUV measurements and modeling, Adv. Space Res., 18, 3

Tobiska, W.K., \& Eparvier, F.G. 1995, Improvements to EUV irradiance modeling in the soft $\mathrm{x}$-rays and FUV, Proceedings of SOLERS22

Tobiska, W.K., Chakrabarti, S., Schmidtke, G., \& Doll, H. 1993, Comparative solar EUV Flux for the San Macro ASSI, Adv. Space Res, 13, 255

White, O.R. 1977, The Solar Output and Its Variation, Colorado Associated Univ. Press, Boulder
Woods, T.N., \& Rottman, G.J. 1997, Solar Lyman- $\alpha$ irradience measuremen ts during two solar cycles, J. Geophys. Res., 102, 8769 